\title{
Women and Alport syndrome
}

\author{
Michelle N. Rheault
}

Received: 7 January 2011 /Revised: 17 February 2011 / Accepted: 18 February 2011 /Published online: 5 March 2011

(C) IPNA 2011

\begin{abstract}
X-linked Alport syndrome (XLAS) is caused by mutations in type IV collagen causing sensorineural hearing loss, eye abnormalities, and progressive kidney dysfunction that results in near universal end-stage renal disease (ESRD) and the need for kidney transplantation in affected males. Until recent decades, the disease burden in heterozygous "carrier" females was largely minimized or ignored. Heterozygous females have widely variable disease outcomes, with some affected females exhibiting normal urinalysis and kidney function, while others develop ESRD and deafness. While the determinants of disease severity in females with XLAS are uncertain, skewing of X-chromosome inactivation has recently been found to play a role. This review will explore the natural history of heterozygous XLAS females, the determinants of disease severity, and the utility of using XLAS females as kidney donors.
\end{abstract}

Keywords X-chromosome inactivation - Glomerular basement membrane $\cdot$ Kidney transplantation · Type IV collagen $\cdot$ Alport syndrome $\cdot$ Familial nephritis

\section{Introduction}

Alport syndrome (AS) is a progressive, inherited disorder of basement membranes that classically presents with

\section{N. Rheault}

Department of Pediatrics,

University of Minnesota Amplatz Children's Hospital,

Minneapolis, MN, USA

\section{N. Rheault ( $\square)$}

Division of Pediatric Nephrology, University of Minnesota, 420 Delaware St. SE, MMC 491,

Minneapolis, MN 55455, USA

e-mail: rheau002@umn.edu microscopic hematuria. Other characteristic features include sensorineural deafness, anterior lenticonus, and progressive kidney dysfunction leading to end-stage renal disease (ESRD) [1]. AS is a relatively common genetic cause of ESRD in the pediatric population, with familial nephritis accounting for $2.3 \%$ of pediatric kidney transplants and $1.9 \%$ of the pediatric dialysis population according to the 2008 report of the North American Pediatric Renal Trials and Collaborative Studies (NAPRTCS) [2].

AS is caused by mutations in type IV collagen, a major constituent of basement membranes [3]. Six isoforms of type IV collagen, $\alpha 1(\mathrm{IV})-\alpha 6(\mathrm{IV})$, encoded by six genes, COL4A1-COL4A6, are variably expressed in basement membranes. These type IV collagen chains self assemble to form specific triple helixes that then form a tertiary network together with laminin, entactin/nidogen, and proteoglycans to ultimately form the basement membranes. The $\alpha 3-\alpha 4-\alpha 5$ (IV) network is the predominant type IV collagen network in the mature glomerular basement membrane (GBM) and certain basement membranes of the cochlea and eye [3]. Mutations in the $\alpha 3, \alpha 4$, or $\alpha 5$ chain of type IV collagen lead to the loss or disruption of the $\alpha 3$ $\alpha 4-\alpha 5$ (IV) network, dysfunction of the affected basement membranes, and the clinical manifestations of AS [1].

There are three common patterns of inheritance in Alport families. About $80 \%$ of AS is inherited in an X-linked manner (XLAS) and caused by mutations in COL4A5 on the $\mathrm{X}$ chromosome. Nearly all affected males will develop ESRD, while heterozygous females exhibit a wide variability in disease severity $[4,5]$. Nearly $90 \%$ of males with XLAS reach ESRD by age 40 years, while only $12 \%$ of heterozygous females do the same [4]. About $15 \%$ of AS is inherited in an autosomal recessive manner (ARAS) due to mutations in both alleles of either COL $4 A 3$ or COL $4 A 4$. Autosomal dominant AS is quite rare (about $5 \%$ of families) 
and is caused by heterozygous mutations in either $C O L 4 A 3$ or COL4A4 [6]. For the remainder of this review, I will focus on heterozygous females with COL4A5 mutations. I will specifically avoid the term "carrier" as this implies a benign or silent phenotype.

Kidney biopsies from heterozygous AS females have a widely variable appearance, with nonspecific findings detected by light microscopy, ranging from a normal appearance to mesangial proliferation to interstitial fibrosis. Electron microscopy may reveal thinning of the GBM early in the disease course; however, the GBM may also have a normal appearance. Later in the disease course, classic thickening and lamellation of the GBM with overlying focal or diffuse podocyte foot process effacement may be evident in severely affected females [1]. Immunostaining for $\alpha 3(\mathrm{IV}), \alpha 4(\mathrm{IV})$, and $\alpha 5(\mathrm{IV})$ in affected males demonstrates the absence of these collagen chains in the GBM in approximately $80 \%$ of patients. In heterozygous females, a mosaic GBM staining pattern is evident in $60-70 \%$ of females due to X-chromosome inactivation (Fig. 1). Skin biopsies have become an accepted method for diagnosing XLAS in order to avoid a more invasive renal biopsy. $\alpha 5$ (IV) along with $\alpha 6$ (IV) are normally present in the epidermal basement membrane (EBM) in the form of $\alpha 5$ $\alpha 5-\alpha 6$ (IV) networks. Immunostaining for $\alpha 5$ (IV) demonstrates its absence from the EBM in most affected males, while a discontinuous staining pattern is apparent in $60-70 \%$ of heterozygous females [1]. One group reported a heterozygous female with normal $\alpha 5$ (IV) immunostaining in the GBM and a discontinuous staining pattern in the EBM [7]. The reason for this discrepancy is unclear but may reflect different $\mathrm{X}$ inactivation patterns in different tissues [8].

\section{Natural history of heterozygous females}

For much of the 20th century, affected females in families with AS were assumed to follow a benign course. Alport himself wrote in 1927 that "the females have deafness and hematuria and live to old age" [9]. Interestingly, the family first reported by Alport included a female with hematuria and deafness who died at 24 years of age. Even in the 1960s investigators believed that "females usually remain well throughout life... and only rarely have women died of the disease" [10]. However, in the 1960s and 1970's case reports about severely affected females started to appear in the literature [11]. In 1985, a report from Hôpital Necker in Paris described the natural history of 36 women from 24 families with hereditary nephritis [12]. One quarter of heterozygous females progressed to ESRD prior to age 35 years, while another $14 \%$ reached end stage after age 45 years. Predictors of progressive kidney disease in this cohort included gross hematuria in childhood, nephrotic syndrome, and diffuse GBM thickening as evidenced by electron microscopy. This was one of the first indications to the renal community that heterozygous females with AS were at significant risk for progression to ESRD.

The most extensive natural history study of heterozygous females was performed by the European Community Alport Syndrome Concerted Action Study and published in 2003 [4]. The investigators characterized 349 heterozygous females from 195 families with proven COL4A5 mutations and compared them to affected hemizygous males within the same families. Microscopic hematuria was found in $95.5 \%$ of XLAS heterozygotes. Thus, although nearly universal in heterozygotes, the absence of microscopic hematuria can not exclude XLAS carrier status. Surprisingly, $75 \%$ of XLAS heterozygotes developed proteinuria. As with other renal diseases, the presence of proteinuria was a significant risk factor for progression to $\operatorname{ESRD}(p<0.001)$. While nearly $90 \%$ of affected males developed ESRD by age 40 years, only $12 \%$ of heterozygous females in this study did the same (Fig. 2). After age 60 years, between $30-40 \%$ of heterozygotes developed ESRD. These data confirmed a substantial burden of kidney disease in this allfemale population, in stark contrast to the previously accepted "benign carrier" designation.
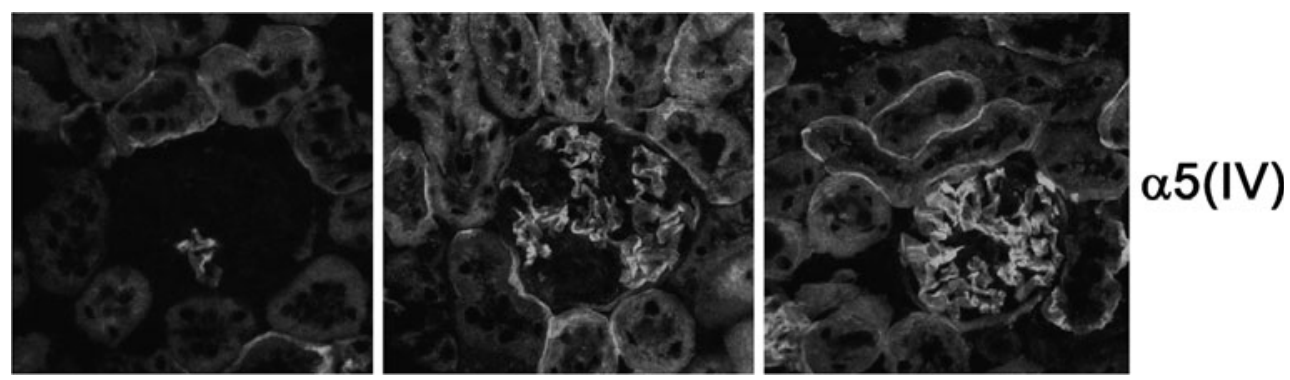

Fig. 1 Glomeruli from a single heterozygous X-linked Alport syndrome (XLAS) mouse demonstrate a wide variability in $\alpha 5$ (IV) expression due to X-inactivation, ranging from nearly absent (left panel) to nearly normal (right panel). Immunohistochemistry was performed as previously published [23] using rat anti- $\alpha 5$ (IV) antibodies (courtesy of Y. Sado). Images courtesy of Y. Segal 


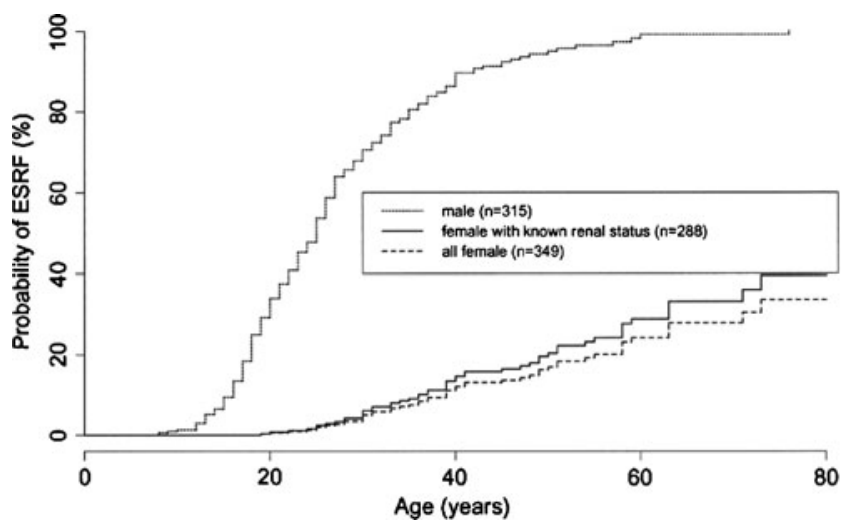

Fig. 2 A natural history study of 195 families with XLAS showed that nearly $90 \%$ of affected males developed end-stage renal disease (ESRD) by age 40 years. In comparison, $12 \%$ of heterozygous females demonstrated ESRD, which increased to $30-40 \%$ after age 60 years. ESRF End-stage renal failure. Reprinted with permission of the American Society of Nephrology (Jais et al.: J Am Soc Nephrol $14: 2603-2610$ [4])

In this same study, hearing loss was detected in $28 \%$ of heterozygous females, with the risk increasing with age [4]. By age 40 years, approximately $90 \%$ of affected males had hearing loss versus $10 \%$ in heterozygous females. The presence of hearing loss in heterozygous females was also a risk factor for progression to $\operatorname{ESRD}(p=0.02)$.

\section{Determinants of disease severity in heterozygous females}

The explanation for the wide variability in outcomes for heterozygous females is uncertain, but likely multifactorial. In affected males there is a significant genotype-phenotype correlation in that nonsense mutations and large deletions are more likely to lead to early ESRD than missense or splice site mutations [5]. In females, these genotypephenotype correlations are not observed [4]. There is also inconsistency in phenotype between females within the same family. Thus, genotype does not seem to be a major determinant of phenotypic heterogeneity in XLAS females.

Over the years, several investigators have suggested that differences in $\mathrm{X}$ chromosome inactivation may influence disease severity in heterozygous females $[13,14]$. $X$ chromosome inactivation is used by mammalian cells to equalize gene dosage between the $\mathrm{XX}$ female and the $\mathrm{XY}$ male [15]. Very early in development, either the maternal or paternal $\mathrm{X}$ chromosome is randomly silenced through a complex cellular machinery [16]. This inactivation choice is passed on to all progeny cells, resulting in a female being a mosaic of cells with either the maternal or paternal $\mathrm{X}$ chromosome active [15]. In a typical female, $50 \%$ of the active X-chromosomes would be of maternal origin while the remaining $50 \%$ would be of paternal origin. However,
$\mathrm{X}$-inactivation ratios can be skewed due to chance, $\mathrm{X}$ chromosome anomalies, X-inactivation modifier genes, such as $X c e$ in the mouse, or mutation selection advantage. $\mathrm{X}$-inactivation has long been thought to be a stable, irreversible phenomenon; however, recent data indicate that some genes can "escape" from X- inactivation [17]. In addition, cells with rapid turnover, such as peripheral blood cells, can demonstrate increased skewing of X-inactivation with age, thought to be due to selection pressure [18].

\section{X-inactivation and Alport females}

Severe skewing of $\mathrm{X}$-inactivation in favor of expression of the mutant COL4A5 has been reported to be responsible for a severe Alport phenotype in two heterozygous females $[19,20]$. The first report was of a woman who reached ESRD by age 30 years and was found to have two separate COL4A5 mutations on the same chromosome [19]. In white blood cells and kidney biopsy tissue, $>90 \%$ of the mRNA messages contained the COL4A5 mutations, suggesting that the mutant $\mathrm{X}$ chromosome was being preferentially expressed. The cause for the severe skewing was not determined, although Turner syndrome (XO) was excluded. In the second report, a heterozygous female with early sensorineural hearing loss and kidney disease was found to have a balanced translocation $\mathrm{t}(\mathrm{X} ; 1)(\mathrm{q} 22.3 ; \mathrm{p} 36.32)$ that disrupted one copy of the COL4A5 gene [20]. Due to the translocation, the normal $\mathrm{X}$ chromosome was preferentially inactivated, leading to a severe phenotype.

Vetrie et al. [13] were unable to find a correlation between $\mathrm{X}$-inactivation measured in lymphocytes and disease severity in a group of 30 heterozygous females and, based on this result, postulated that lymphocyte $\mathrm{X}$ inactivation ratios do not accurately reflect $\mathrm{X}$-inactivation ratios in renal tissue. This possibility is supported by reports showing that severe skewing of $\mathrm{X}$ inactivation $(>90: 10)$ in lymphocytes increases with age, likely due to selection pressure on peripheral blood cells that rapidly turn over [18]. Additionally, skewed patterns of X inactivation are more likely to be found in the blood than in other tissues, such as buccal and urinary epithelia [18].

Two groups have attempted to use $\alpha 5$ (IV) expression in the EBM as revealed by immunofluorescence microscopy as a surrogate for $\mathrm{X}$ inactivation and $\alpha 5$ (IV) expression in the GBM-with conflicting results. Nakanishi et al. found a highly significant negative correlation between EBM $\alpha 5$ (IV) expression and urine protein to creatinine ratio in 25 heterozygous females [21]. Masella et al. found no correlation at all in a study of 22 heterozygous females using similar methodology [22]. Therefore, it is currently impossible to accurately predict renal outcomes in females based on measurements of $\alpha 5$ (IV) expression in the skin. 


\section{Lessons learned from $X$ inactivation studies in murine XLAS}

The availability of a transgenic mouse model of XLAS has allowed us to directly test the influence of skewed X inactivation ratios on disease outcomes. The XLAS mouse recapitulates the classic pathological findings of human AS, including the ultrastructural and $\alpha 5$ (IV) immunostaining findings [23]. Affected male mice develop increasing proteinuria and azotemia with age and have an average lifespan of 23 weeks [23]. Heterozygous females have a less severe but widely variable phenotype, with an average lifespan of 39 weeks.

In mice, the choice of which $\mathrm{X}$ chromosome is inactivated is influenced by the $\mathrm{X}$ controlling element (Xce) on the X chromosome [24]. Three Xce alleles have been identified: $X c e^{a}, X c e^{b}$, and $X c e^{c}$ [24]. Random X inactivation is observed in mice homozygous for Xce alleles, whereas mice heterozygous for Xce alleles will preferentially inactivate $X c e^{a}>X c e^{b}>X c e^{c}$. Inbred XLAS mice on a C57BL6/J background possess the $X c e^{b}$ allele and would be expected to demonstrate random (50:50) X inactivation. By crossing heterozygous female XLAS mice with two strains of $129 \mathrm{SvPas}$ mice, we generated two groups of heterozygous female mice that differed only at the Xce locus [25]. In group $1\left(X c e^{a / b}\right)$, expression of the mutant Col4a 5 allele was favored, while in group $2\left(X c e^{b / c}\right)$ expression of the normal Col4a5 allele was favored. Whole kidney mRNA expression assays confirmed that the two groups diverged in $\mathrm{X}$ inactivation ratios, with group 1 demonstrating an average ratio of 53:47 (mutant:wild-type) and group 2 demonstrating a ratio of 46:54 [25]. Despite this modest skewing of $\mathrm{X}$ inactivation ratios overall, there was a distinct survival advantage in mice in group 2 . In addition, there was a positive correlation between $\mathrm{X}$ inactivation ratio and both urine protein excretion and plasma urea nitrogen levels at 6 months of age [25]. This study was the first to confirm a direct effect of Xinactivation skewing on the XLAS heterozygous female phenotypes. However, X inactivation ratios only explained a portion of the observed variability in outcomes. Other determinants of disease progression in heterozygous females remain to be discovered.

The applicability of these findings to the prediction of human XLAS heterozygote outcomes is unclear. Humans do not have an Xce locus like the mouse; however, other genes or chromosomal regions are likely to play a similar role. As discussed above, whole blood lymphocyte X inactivation ratios may not reflect the state in the kidney. Unfortunately, it is not possible to perform whole kidney mRNA assays to evaluate $\mathrm{X}$ inactivation ratios in humans. Consequently, we are currently unable to predict disease outcomes in heterozygous human females from measure- ments of $\mathrm{X}$ inactivation ratios. Further research into methods for predicting individual outcomes is needed.

\section{XLAS heterozygotes as kidney donors}

Female heterozygotes are often called upon to be kidney donors for their affected sons or other family members. Mothers often feel significant guilt for passing the mutant COL4A5 gene on to their sons, while simultaneously feeling a moral responsibility to help their sons in any way they can, particularly by donating their own kidney $[26,27]$. This presents the clinician with the difficult task of balancing the risk to the donor with the benefits to the recipient [27]. There is little long-term data regarding outcomes in either the donors or recipients in this situation. One report from Germany described five XLAS and one ARAS heterozygotes who served as kidney donors [26]. One donor had proteinuria prior to transplant and all had microscopic hematuria. Three donors developed new onset hypertension and two developed new proteinuria, while renal function declined by $25-60 \%$ over a period of 2 14 years post-transplant in four of the donors [26]. None of the donors developed ESRD. For the recipients, there was $100 \%$ graft survival at 1 and 5 years, while one kidney failed after 10 years.

It is recommended that Alport heterozygotes be kidney donors of last resort [27]. Heterozygous females with proteinuria or hearing loss should be excluded from consideration as kidney donors. Heterozygous females with isolated microscopic hematuria, normal kidney function, normal blood pressure, and normal hearing can be considered only after careful counseling about risks. Research into long-term outcomes and the utility of renal protective methods for donors, such as angiotensin converting enzyme inhibition, is needed.

\section{Conclusions}

There is wide phenotypic variability in disease expression in heterozygous XLAS females. Heterozygous females with XLAS are at risk of developing ESRD and sensorineural hearing loss, although at lower rates than affected males. Heterozygous XLAS females should not be considered to be benign carriers, but rather as women at risk for developing disease. They should be followed clinically in a similar manner to affected males to evaluate for early signs of progression. Proteinuria and hearing loss are predictors of progression to ESRD. When tested directly in controlled genetic backgrounds, favorable X-inactivation increases survival and improves clinical parameters in heterozygous XLAS female mice. Further research is required 
to determine methods to predict individual outcome for women with XLAS.

Acknowledgments Thank you to Dr. Clifford Kashtan for helpful comments on the manuscript and Dr. Yoav Segal for providing images of the heterozygous XLAS mouse.

\section{Review Questions (answers are given following the references)}

1. The percentage of Alport syndrome inherited in an Xlinked manner is:
a. $100 \%$
b. $80 \%$
c. $50 \%$
d. $20 \%$

2. Heterozygous XLAS females can present with:
a. Normal urinalysis
b. Microscopic hematuria
c. Proteinuria
d. Sensorineural hearing loss
e. All of the above

3. The following has been shown to influence disease outcome in XLAS heterozygous females
a. $\mathrm{X}$ inactivation
b. Ethnicity
c. ACE inhibition
d. Pregnancy
e. Genotype

4. Which of the following are risk factors for renal disease progression in XLAS heterozygous females?
a. Microscopic hematuria
b. Proteinuria
c. Use of hormone replacement therapy
d. Hearing loss
e. B and D
f. B and $\mathrm{C}$

5. $\mathrm{X}$ inactivation patterns in this tissue can predict $\mathrm{X}$ inactivation patterns in the kidney
a. Blood lymphocytes
b. Skin
c. Urinary epithelium
d. None of the above

6. Heterozygous XLAS females should be considered as kidney donors if they meet all of the following criteria except:
a. Presence of microscopic hematuria
b. Presence of proteinuria
c. Absence of sensorineural hearing loss
d. Absence of hypertension

\section{References}

1. Kashtan CE (2009) Familial hematuria. Pediatr Nephrol 24:19511958

2. North American Pediatric Renal Transplant Cooperative Study (NAPRTCS) (2008) North American Pediatric Renal Transplant Cooperative Study Annual Report 2008. NAPRTCS, Boston

3. Miner JH (1999) Renal basement membrane components. Kidney Int 56:2016-2024

4. Jais JP, Knebelmann B, Giatras I, De Marchi M, Rizzoni G, Renieri A, Weber M, Gross O, Netzer KO, Flinter F, Pirson Y, Dahan K, Wieslander J, Persson U, Tryggvason K, Martin P, Hertz JM, Schroder C, Sanak M, Carvalho MF, Saus J, Antignac C, Smeets H, Gubler MC (2003) X-linked Alport syndrome: natural history and genotype-phenotype correlations in girls and women belonging to 195 families: a "European Community Alport Syndrome Concerted Action" study. J Am Soc Nephrol 14:2603-2610

5. Jais JP, Knebelmann B, Giatras I, De Marchi M, Rizzoni G, Renieri A, Weber M, Gross O, Netzer KO, Flinter F, Pirson Y, Verellen C, Wieslander J, Persson U, Tryggvason K, Martin P, Hertz JM, Schroder C, Sanak M, Krejcova S, Carvalho MF, Saus J, Antignac C, Smeets H, Gubler MC (2000) X-linked Alport syndrome: natural history in 195 families and genotype- phenotype correlations in males. J Am Soc Nephrol 11:649-657

6. Marcocci E, Uliana V, Bruttini M, Artuso R, Silengo MC, Zerial M, Bergesio F, Amoroso A, Savoldi S, Pennesi M, Giachino D, Rombola G, Fogazzi GB, Rosatelli C, Martinhago CD, Carmellini M, Mancini R, Di Costanzo G, Longo I, Renieri A, Mari F (2009) Autosomal dominant Alport syndrome: molecular analysis of the COL4A4 gene and clinical outcome. Nephrol Dial Transplant 24:1464-1471

7. Hamiwka LA, George DH, Grisaru S, Midgley JP (2007) Discordance between skin biopsy and kidney biopsy in an Xlinked carrier of Alport syndrome. Pediatr Nephrol 22:1050-1053

8. Gale RE, Wheadon H, Boulos P, Linch DC (1994) Tissue specificity of X-chromosome inactivation patterns. Blood 83:2899-2905

9. Alport AC (1927) Hereditary Familial Congenital Haemorrhagic Nephritis. Br Med J 1:504-506

10. Perkoff GT (1964) Familial aspects of diffuse renal diseases. Annu Rev Med 15:115-124

11. Albert MS, Leeming JM, Wigger HJ (1969) Familial nephritis associated with the nephrotic syndrome. In a family with severe involvement in females. Am J Dis Child 117:153-155

12. Grunfeld JP, Noel LH, Hafez S, Droz D (1985) Renal prognosis in women with hereditary nephritis. Clin Nephrol 23:267-271

13. Vetrie D, Flinter F, Bobrow M, Harris A (1992) X inactivation patterns in females with Alport's syndrome: a means of selecting against a deleterious gene? J Med Genet 29:663-666

14. Shimizu Y, Nagata M, Usui J, Hirayama K, Yoh K, Yamagata K, Kobayashi M, Koyama A (2006) Tissue-specific distribution of an alternatively spliced COL4A5 isoform and non-random $\mathrm{X}$ chromosome inactivation reflect phenotypic variation in heterozygous X-linked Alport syndrome. Nephrol Dial Transplant 21:1582-1587

15. Lyon MF (2002) X-chromosome inactivation and human genetic disease. Acta Paediatr Suppl 91:107-112

16. Ng K, Pullirsch D, Leeb M, Wutz A (2007) Xist and the order of silencing. EMBO Rep 8:34-39

17. Berletch JB, Yang F, Disteche CM (2010) Escape from X inactivation in mice and humans. Genome Biol 11:213

18. Sharp A, Robinson D, Jacobs P (2000) Age- and tissue-specific variation of $\mathrm{X}$ chromosome inactivation ratios in normal women. Hum Genet 107:343-349 
19. Guo C, Van Damme B, Vanrenterghem Y, Devriendt K, Cassiman JJ, Marynen P (1995) Severe alport phenotype in a woman with two missense mutations in the same COL4A5 gene and preponderant inactivation of the $\mathrm{X}$ chromosome carrying the normal allele. J Clin Invest 95:1832-1837

20. Iijima K, Nozu K, Kamei K, Nakayama M, Ito S, Matsuoka K, Ogata T, Kaito H, Nakanishi K, Matsuo M (2010) Severe Alport syndrome in a young woman caused by a $\mathrm{t}(\mathrm{X} ; 1)(\mathrm{q} 22.3 ; \mathrm{p} 36.32)$ balanced translocation. Pediatr Nephrol 25:2165-2170

21. Nakanishi K, Iijima K, Kuroda N, Inoue Y, Sado Y, Nakamura H, Yoshikawa N (1998) Comparison of alpha5(IV) collagen chain expression in skin with disease severity in women with X-linked Alport syndrome. J Am Soc Nephrol 9:1433-1440

22. Massella L, Onetti Muda A, Faraggiana T, Bette C, Renieri A, Rizzoni G (2003) Epidermal basement membrane alpha 5(IV) expression in females with Alport syndrome and severity of renal disease. Kidney Int 64:1787-1791

23. Rheault MN, Kren SM, Thielen BK, Mesa HA, Crosson JT, Thomas W, Sado Y, Kashtan CE, Segal Y (2004) Mouse model of X-linked Alport syndrome. J Am Soc Nephrol 15:1466-1474

24. Chadwick LH, Pertz LM, Broman KW, Bartolomei MS, Willard HF (2006) Genetic control of X chromosome inactivation in mice: definition of the Xce candidate interval. Genetics 173:2103-2110
25. Rheault MN, Kren SM, Hartich LA, Wall M, Thomas W, Mesa HA, Avner P, Lees GE, Kashtan CE, Segal Y (2010) Xinactivation modifies disease severity in female carriers of murine X-linked Alport syndrome. Nephrol Dial Transplant 25:764-769

26. Gross O, Weber M, Fries JW, Muller GA (2009) Living donor kidney transplantation from relatives with mild urinary abnormalities in Alport syndrome: long-term risk, benefit and outcome. Nephrol Dial Transplant 24:1626-1630

27. Kashtan CE (2009) Women with Alport syndrome: risks and rewards of kidney donation. Nephrol Dial Transplant 24:13691370

\section{Answers}

1) $b$

2) e

3) a

4) e

5) $d$

6) $b$ 